\section{Case Reports in Neurology}

Case Rep Neurol 2020;12:276-281

DOI: 10.1159/000508404

Published online: September 17, 2020

(C) 2020 The Author(s)

Published by S. Karger AG, Basel www.karger.com/crn

This article is licensed under the Creative Commons Attribution-NonCommercial 4.0 International License (CC BY-NC) (http://www.karger.com/Services/OpenAccessLicense). Usage and distribution for commercial purposes requires written permission.

\title{
Isolated Cervical Myelitis in Lyme Disease: A Rare Manifestation of Acute Neuroborreliosis
}

\author{
Maren Hieber Johann Lambeck Sebastian Rauer Juergen Bardutzky \\ Clinic of Neurology and Neurophysiology, Medical Center - University of Freiburg, Faculty \\ of Medicine, University of Freiburg, Freiburg, Germany
}

\section{Keywords}

Lyme disease $\cdot$ Neuroborreliosis $\cdot$ Myelitis

\begin{abstract}
Neuroborreliosis is the neurological manifestation of Lyme disease, a tick-borne infectious multi-system disease caused by Borrelia burgdorferi sensu lato. It appears in 3 to $15 \%$ of all cases of acute Lyme disease, and includes meningitis, cranial neuritis, and painful radiculoneuritis as the most common manifestations. We report a case of acute neuroborreliosis that manifested as extended isolated cervical myelitis. Not only the manifestation as isolated myelitis in the early stages of borreliosis represents a rarity, but also the strong contrast between mild clinical symptoms and pronounced imaging findings in this case is remarkable.
\end{abstract}

(C) 2020 The Author(s)

Published by S. Karger AG, Basel

\section{Introduction}

Neuroborreliosis is the neurological manifestation of Lyme disease, a tick-borne infectious multi-system disease caused by the spirochete Borrelia burgdorferi sensu lato. Neurological symptoms represent the second (Germany) and third (United States) most common manifestation of acute Lyme disease (about 3-15\%) [1, 2], while the cutaneous manifestation of erythema migrans (about $80 \%$ ) typically is the earliest and most common clinical symptom. 


\section{Case Reports in Neurology}

Case Rep Neurol 2020;12:276-281

DOI: 10.1159/000508404

Hieber et al.: Isolated Cervical Myelitis in Lyme Disease

Typical neurological manifestations usually appear weeks to months after the tick bite. They include meningitis, cranial neuritis (especially facial nerve palsy), and painful radiculoneuritis. Although parenchymal inflammation of the brain and the spinal cord (i.e., encephalitis, myelitis, or a combination of both) has been described, it represents an extremely rare manifestation of Lyme disease in the earlier stages of the disease (around $4 \%$ of all cases with nervous system affection), especially in adults [3]. The rare late form of neuroborreliosis ("chronic neuroborreliosis"), which appears months to years after infection typically manifests as encephalomyelitis with spastic-atactic gait and bladder dysfunction [3].

We report a case of early extensive cervical myelitis due to infection with $B$. burgdorferi as a rare manifestation of acute neuroborreliosis.

\section{Case Presentation}

A 45-year-old woman was admitted to an external secondary care hospital due to pronounced cervicobrachialgia. As potential cause she had reported a bicycle accident 3 weeks prior to admission that had been followed by nuchal pain lasting for 2 days. Following a temporary pain-free period of about 8 days, the symptoms had returned again, this time accompanied by sub-febrile temperatures, nausea, and oscillopsia. Due to the reported bicycle accident, the pronounced cervicobrachialgia had initially been considered to be posttraumatic by the external treating physicians. Administration of intravenous analgesia had been started, resulting in partial remission of the nuchal pain. Due to the pronounced drug-resistant nuchal pain, a cervical MRI was performed in the secondary care hospital, which showed an edema with central intramedullary hyperintensity in the T2-weighed sequences in the cervical spinal cord (Fig. 1). This finding led the treating physicians to discard their initial hypothesis of posttraumatic cervicobrachialgia and caused the transfer to our tertiary medical center (neurologic department). Here, the patient reported the above-mentioned course of symptoms (for a graphic presentation of the course, see Fig. 2). Besides allergic asthma, several food intolerances and malaria as a child (when she had lived in Africa), the patient did not report any preexisting illnesses. During her stay in the secondary care hospital, an extended analgetic medication with metamizole, diclofenac and morphine, accompanied by pantoprazole and dimenhydrinate was established. Further pre-existing medication was denied by the patient. The patient was married, had one child, and worked in the IT sector. She reported neither pets nor further animal contact nor any recent travel.

Clinical examination upon arrival at our department revealed subtle neck stiffness, which was judged to be of musculoskeletal origin rather than due to meningeal inflammation. Additionally, Kernig's and Brudzinski's signs remained negative. During clinical examination, the patient stated diffuse numbness of the right arm without dermatomal correlation with one or more cervical nerve roots or a peripheral nerve, as well as slight weakness of the right arm. Neither numbness nor weakness could be objectified by respective clinical testing. Further symptoms, including autonomic symptoms such as urinary and fecal retention, were neither reported by the patient nor found during clinical examination.

Initial basic blood parameters (blood count, electrolytes, retention, infection, hepatic, and coagulation parameters) did not show any abnormalities. Further blood workup as well as an urinalysis, which was additionally performed later, did not reveal remarkable findings except a deficit of folic acid.

Lumbar puncture was performed immediately upon transfer and revealed lymphocytic pleocytosis $(1,404 / \mu \mathrm{L}$, normal $<5 / \mu \mathrm{L})$, and elevation of both total protein $(978 \mathrm{mg} / \mathrm{L}$, normal 


\section{Case Reports in Neurology}

Case Rep Neurol 2020;12:276-281

DOI: $10.1159 / 000508404$

(c) 2020 The Author(s). Published by S. Karger AG, Basel www.karger.com/crn

Hieber et al.: Isolated Cervical Myelitis in Lyme Disease

$<450 \mathrm{mg} / \mathrm{L}$ ), and lactate ( $4.1 \mathrm{mmol} / \mathrm{L}$, normal 1.1-2.1 mmol/L). Because of the above-mentioned oscillopsia reported by the patient, cranial MRI was complemented but revealed unremarkable findings. Cervical MRI was repeated in-house, this time with contrast-enhancement, and revealed an unaltered edema state in the cervical medulla with central hyperintensity in T2-weighted sequences. Only subtle intramedullary contrast enhancement was detected at the level of vertebral body C7 (Fig. 1).

Due to the combination of MRI findings and lymphocytic pleocytosis infectious myelitis of unknown origin was suspected, and empiric anti-infective treatment with acyclovir $750 \mathrm{mg}$ three times a day, ceftriaxone $2 \mathrm{~g}$ once a day, and ampicillin $5 \mathrm{~g}$ three times a day was started immediately.

The extended diagnostic workup detected Borrelia-specific antibodies in serum (by ELISA: IgM 6.98 [normal <5], IgG 43.07 [normal <16]) and proved intrathecal synthesis of Borrelia-specific IgM and IgG in ELISA by positive antibody specificity indices for IgM (4.10 [normal <1.5]) and IgG (68.2 [normal <1.5]), thus confirming acute neuroborreliosis. Furthermore, Western blot analysis confirmed Borrelia-specific IgM in serum, while Borrelia-specific IgG were not detectable by Western blot. Polymerase chain reaction (PCR) for B. burgdorferi sensu lato was positive in the first cerebrospinal fluid (CSF) analysis taken at admission but negative in the second CSF sample taken 2 days later. Further extensive microbiologic, virologic, and immunologic testing (for tuberculosis, Lues, listeria, toxoplasmosis, mycoplasma pneumoniae, chlamydiae, legionella, fungi, HSV, VZV, rubella virus, FSME, HIV, autoimmune encephalitis antibodies, antineuronal antibodies, antibodies against MOG and aquaporine 4, oligoclonal bands, and blood cultures) remained negative in both blood and CSF samples. In line with a diagnosis of borreliosis and on specific inquiry only, the patient reported to have suffered from an axillary erythema migrans approximately 14 days prior to admission. The patient was clinically stable, and the cervicobrachialgia was treated with paracetamol $500 \mathrm{mg}$ four times per day. Since the pain described by the patient was fitting with neuropathic pain, medication with amitriptyline $25 \mathrm{mg}$ twice a day was initiated additionally. Antibiotic therapy with parenteral ceftriaxone $2 \mathrm{~g}$ per day was continued for 3 weeks.

\section{Discussion and Conclusion}

We present a case of acute neuroborreliosis that clinically manifested as myelitis. While neurological manifestation is seen in $3-15 \%$ of all cases of acute Lyme disease and is typically characterized by meningitis, radiculoneuritis, and/or cranial neuritis, our patient lacked all these typical manifestations and their corresponding symptoms (headache, distinctive nuchal rigidity, back pain with nocturnal worsening, and - often bilateral - facial nerve palsy). In a German retrospective collective of patients with neuroborreliosis, myelitis was found in only 5 out of 68 patients. All of these 5 patients showed more severe symptoms with paraspasticity $(4 / 5)$, leg weakness $(2 / 5)$, or arm weakness $(1 / 5)$. By comparison to our patient, these patients in parts presented later, with a duration of symptoms between 23 and 733 days, thereby representing at least in parts a manifestation of chronic neuroborreliosis [4]. A Swedish retrospective study reported isolated myelitis in 5 out of 141 patients with neuroborreliosis [5] without further description of clinical severity. Several case reports describe the manifestation of acute neuroborreliosis by myelitis [6-8], but the majority is on children. A case of an adult patient with acute transverse myelitis as manifestation of acute Lyme disease has recently been reported by Dumic et al. [9]. Their patient presented with severe symptoms of motor and sensory deficits of the legs as well as urinary retention and constipation. While 


\section{Case Reports in Neurology}

Case Rep Neurol 2020;12:276-281

DOI: $10.1159 / 000508404$ www.karger.com/crn

Hieber et al.: Isolated Cervical Myelitis in Lyme Disease

showing similar pronounced neuroimaging features, our case stands out by our patient's extremely mild clinical symptoms of minimal sensorimotor deficits of the right arm, and slight neck pain. We found only one further case reported by Bigi et al. [7] on a 12-year-old boy showing similar disparity between distinct imaging results and mild clinical presentation.

Although neurological symptoms were atypical and only mild, the criteria for acute neuroborreliosis as described in German [10] as well as European [11] and American [12] guidelines were fulfilled (i.e., disease-typical imaging results, proof of CSF pleocytosis, intrathecal synthesis of borrelia-specific antibodies, and the additionally positive PCR). Regarding the fulfillment of diagnostic criteria, atypical clinical symptoms as in our case are also of formal interest, since both German and European guidelines actually require a "typical clinical picture" or "neurological symptoms suggestive of neuroborreliosis" $[10,11]$ for the definite diagnosis of neuroborreliosis.

While typical clinical signs often lead the treating physician to the correct diagnosis, in the present case it was the imaging result of spinal cord edema in combination with inflammatory CSF findings suggestive of an inflammable process that led to the suspicion of myelitis of infectious origin. The typical pathogen spectrum of infectious myelitis spans HSV, VZV, HIV, EBV, and CMV. Rarer pathogens include tick-borne encephalitis, mycoplasma pneumoniae, borrelia, mycobacteria tuberculosis, and treponema pallidum. Single case reports describe listeria monocytogenes-related myelitis [13]. Due to suspected infectious origin, we started a broad empiric anti-infective treatment with acyclovir, ceftriaxone, and ampicillin in analogy to well-known empiric treatment in meningitis/meningo-encephalitis. After PCR for HSV and VZV had returned negative, acyclovir was stopped. Treatment was further reduced to ceftriaxone monotherapy after $B$. burgdorferi had been confirmed as the specific infectious etiology.

The patient was only asked directly about whether a tick bite and the subsequent cutaneous symptoms had occurred following proof of acute neuroborreliosis. If this question had been asked earlier, the possibility of neuroborreliosis as a differential diagnosis would have also been considered earlier. This underlines the importance of taking a thorough patient history. On the other hand, the bicycle accident that was initially postulated as the causative event turned out to be a confounder.

Besides an infectious etiology, acute myelitis can also be of postinfectious/autoimmune etiology, and - in these cases - is treated with high-dose corticosteroids, immunoglobulin therapy or plasmapheresis. In this case, and supported by the mild clinical symptoms of our patient, we followed a concept of broad anti-infective and symptomatic analgetic treatment only. Other authors report the additional early application of steroids in combination with the anti-infective therapy to potentially have favourable effects [14].

\section{Statement of Ethics}

Written informed consent has been obtained from the patient for publication of the case, including images.

\section{Conflict of Interest Statement}

The authors have no conflicts of interest to declare. 


\section{Case Reports in Neurology}

\begin{tabular}{l|l}
\hline Case Rep Neurol 2020;12:276-281 \\
\hline DOI: 10.1159/000508404 & $\begin{array}{l}\text { @ 2020 The Author(s). Published by S. Karger AG, Basel } \\
\text { www.karger.com/crn }\end{array}$ \\
\hline
\end{tabular}

Hieber et al.: Isolated Cervical Myelitis in Lyme Disease

\section{Funding Sources}

The authors did not receive any funding.

\section{Author Contributions}

M.H.: patient care, concept and design, acquisition and interpretation of data, drafting, revision, and final approval of the manuscript. J.L.: patient care, interpretation of data, critical revision, and final approval of the manuscript. S.R.: patient care, critical revision, and final approval of the manuscript. J.B.: patient care, concept and design, interpretation of data, critical revision, and final approval of the manuscript.

\section{References}

1 Steere AC, Sikand VK. The presenting manifestations of Lyme disease and the outcomes of treatment. N Engl J Med. 2003 Jun;348(24):2472-4.

2 Wilking H, Stark K. Trends in surveillance data of human Lyme borreliosis from six federal states in eastern Germany, 2009-2012. Ticks Tick Borne Dis. 2014 Apr;5(3):219-24.

3 Hansen K, Lebech AM. The clinical and epidemiological profile of Lyme neuroborreliosis in Denmark 19851990. A prospective study of 187 patients with Borrelia burgdorferi specific intrathecal antibody production. Brain. 1992 Apr;115(Pt 2):399-423.

4 Schwenkenbecher P, Pul R, Wurster U, Conzen J, Pars K, Hartmann H, et al. Common and uncommon neurological manifestations of neuroborreliosis leading to hospitalization. BMC Infect Dis. 2017 Jan;17(1):90.

5 Bremell D, Dotevall L. Oral doxycycline for Lyme neuroborreliosis with symptoms of encephalitis, myelitis, vasculitis or intracranial hypertension. Eur J Neurol. 2014 Sep;21(9):1162-7.

6 Meurs L, Labeye D, Declercq I, Piéret F, Gille M. Acute transverse myelitis as a main manifestation of early stage II neuroborreliosis in two patients. Eur Neurol. 2004;52(3):186-8.

7 Bigi S, Aebi C, Nauer C, Bigler S, Steinlin M. Acute transverse myelitis in Lyme neuroborreliosis. Infection. 2010 Oct;38(5):413-6.

8 Blassnig-Ezeh A, Schober H, Luetschg J, Jäger A, Simma B. Two cases of unusual Lyme infection presenting as central nervous system Lyme disease. Klin Padiatr. 2013 Mar;225(2):91-2.

9 Dumic I, Vitorovic D, Spritzer S, Sviggum E, Patel J, Ramanan P. Acute transverse myelitis - A rare clinical manifestation of Lyme neuroborreliosis. IDCases. 2018 Dec;15:e00479.

10 Rauer S, Kastenbauer S, Fingerle V, Hunfeld KP, Huppertz HI, Dersch R. Lyme Neuroborreliosis. Dtsch Arztebl Int. 2018 Nov;115(45):751-6.

11 Mygland A, Ljøstad U, Fingerle V, Rupprecht T, Schmutzhard E, Steiner I. EFNS guidelines on the diagnosis and management of European Lyme neuroborreliosis. Eur J Neurol. 2010 Jan;17(1):8-16, e1-4.

12 Appendix C: practice parameter: diagnosis of patients with nervous system lyme borreliosis (lyme disease). Continuum (Minneap Minn). 2015;21(6 Neuroinfectious Disease):1770-2.

13 Castro A, Hernández OH, Uribe CS, Guerra A, Urueña P. [Brainstem encephalitis and myelitis due to Listeria monocytogenes: a case report and literature review]. Biomedica. 2013 Jul-Sep;33(3):343-9.

14 Kaiser EA, George DK, Rubenstein MN, Berger JR. Lyme myelopathy: case report and literature review of a rare but treatable disorder. Mult Scler Relat Disord. 2019 Apr;29:1-6. 


\section{Case Reports in Neurology}

\begin{tabular}{l|l}
\hline Case Rep Neurol 2020;12:276-281 \\
\hline DOI: 10.1159/000508404 & $\begin{array}{l}\text { (c) 2020 The Author(s). Published by S. Karger AG, Basel } \\
\text { www.karger.com/crn }\end{array}$ \\
\hline
\end{tabular}

Hieber et al.: Isolated Cervical Myelitis in Lyme Disease
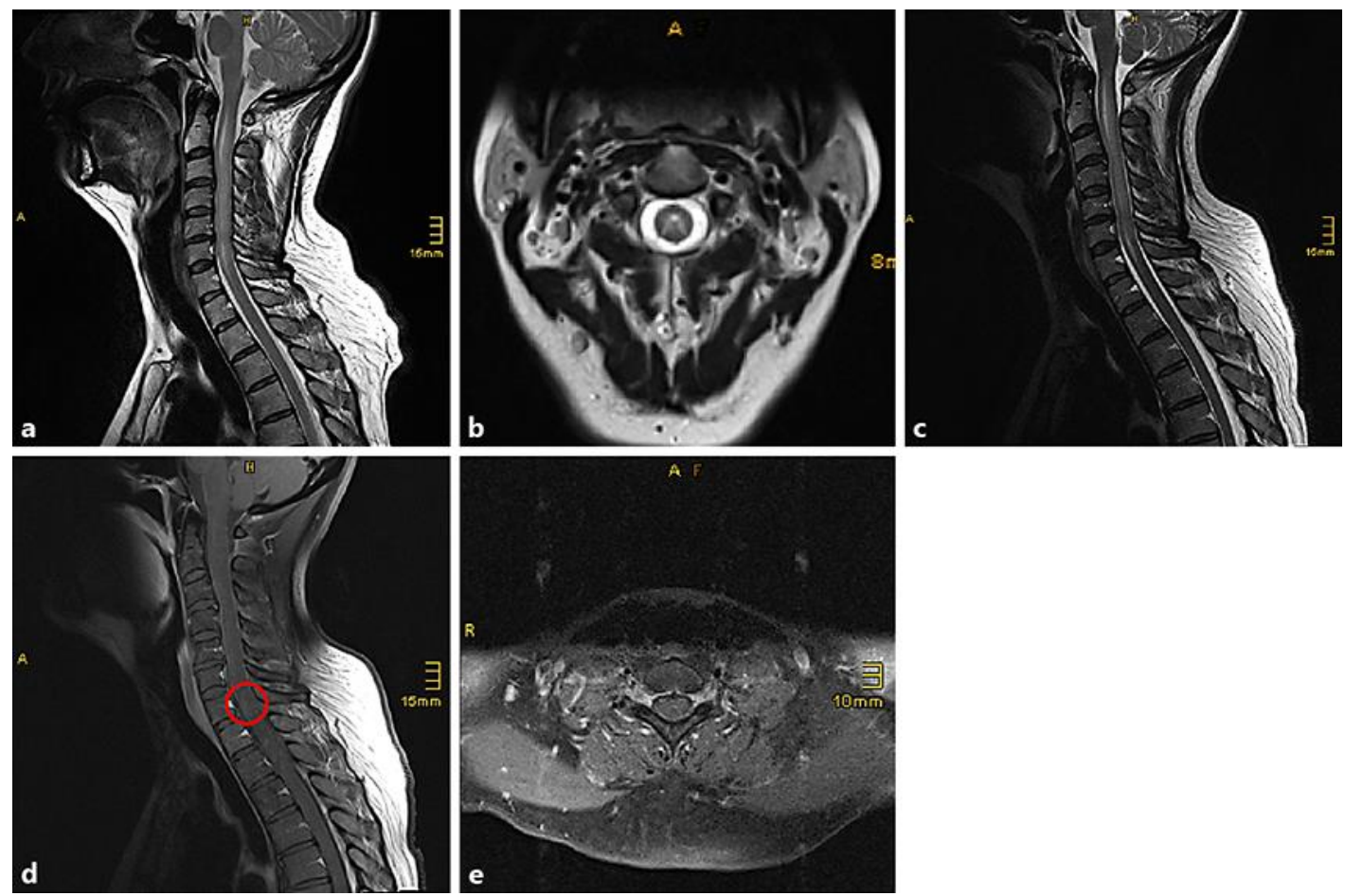

Fig. 1. Cervical MRI was performed externally due to pronounced cervicobrachialgia and revealed edema in the cervical spinal cord in T2-weighted imaging $(\mathbf{a}, \mathbf{b})$. The repeated inhouse cervical MRI revealed stable findings of cervical edema of the spinal cord (c). After gadolinium injection it showed subtle contrast enhancement at the level of vertebral body C7 (red circle in d; e).

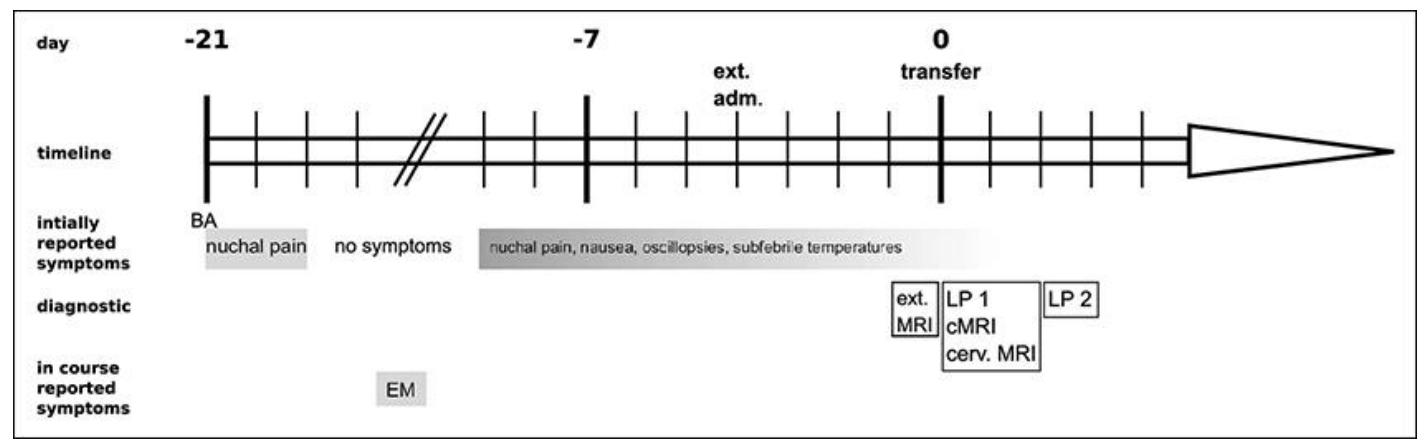

Fig. 2. This graphical timeline shows the course of the reported symptoms as well as the timepoints of external admission (ext. adm.), transfer to our department (transfer), and of the most important diagnostic procedures: the external cervical MRI (ext. MRI) that showed the cervical myelopathy, the in-house cerebral MRI (cMRI) that revealed unremarkable findings, the in-house repeated contrast-enhanced cervical MRI (cerv. MRI), and the two lumbal punctures (LP1, LP2) showing lymphocytic pleocytosis and proofing intrathecal synthesis of Borrelia-specific antibodies. Notably, the patient reported the preceding erythema migrans (EM) on specific inquiry only and upon already secured diagnosis. In contrast, the spontaneously reported preceding bicycle accident (BA) turned out to be a confounder. Neither at the timepoint of initial clinical admission nor upon repeated local examination after the patient's specific statement the reported erythema migrans was visible. 